\section{Subacute sclerosing panencephalitis still occurring in South Africa: Clinicians need to remain vigilant}

To the Editor: We report on another child with subacute sclerosing panencephalitis (SSPE), expanding the case series reported by Kija et al. ${ }^{[1]}$ to six affected children from the Western Cape region of South Africa (SA) diagnosed in the past 18 months.

The child presented to Red Cross War Memorial Children's Hospital, Cape Town, at 6 years and 3 months of age. She had recently returned to SA after living for several years in Zimbabwe. Her caregivers in SA had not seen her regularly, but understood that her previous health and development had been normal. She was admitted with a reported 1-day history of suddenonset weakness and abnormal movements. She had no known history of toxin exposure or prodromal symptoms prior to her presentation. She had neurodevelopmental regression and demonstrated emotional lability with incoherent speech and disorientation. She was not independently mobile. Her tone and power were normal and symmetrical with brisk deep tendon reflexes. Her abnormal movements were noted to be myoclonic jerks and atonic seizures which manifested as 'head nods. She was systemically well with no evidence of sepsis, and while HIV-exposed she had tested negative. Cerebrospinal fluid (CSF) microscopy and chemistry results were normal. An electroencephalogram (EEG) was initially slow with no epileptiform activity correlating with the jerks. Owing to concern about her dramatic presentation and apparent acute-onset encephalopathy, an emergency magnetic resonance imaging (MRI) scan of the brain was performed. This showed high signal on $\mathrm{T} 2$ and fluid attenuation inversion recovery (FLAIR) in the posterosuperior putamina, seen to a greater extent on the left than the right (Fig. 1). On the basis of this finding and the unusual clinical phenotype, SSPE was suspected. The child's CSF measles IgG was positive, while CSF measles polymerase chain reaction (PCR) tested negative.

The history of prior measles infection was not initially elicited from the main caregiver, but on further questioning it emerged that the child had contracted measles during the epidemic of 2009 while in SA. The further history unfolded that she had returned from Zimbabwe several months previously but into the care of a different family member. At this point it was confirmed that her symptoms were more chronic and she had been managed at Tygerberg Children's Hospital, where SSPE had been diagnosed previously. The caregiver at the time was counselled regarding

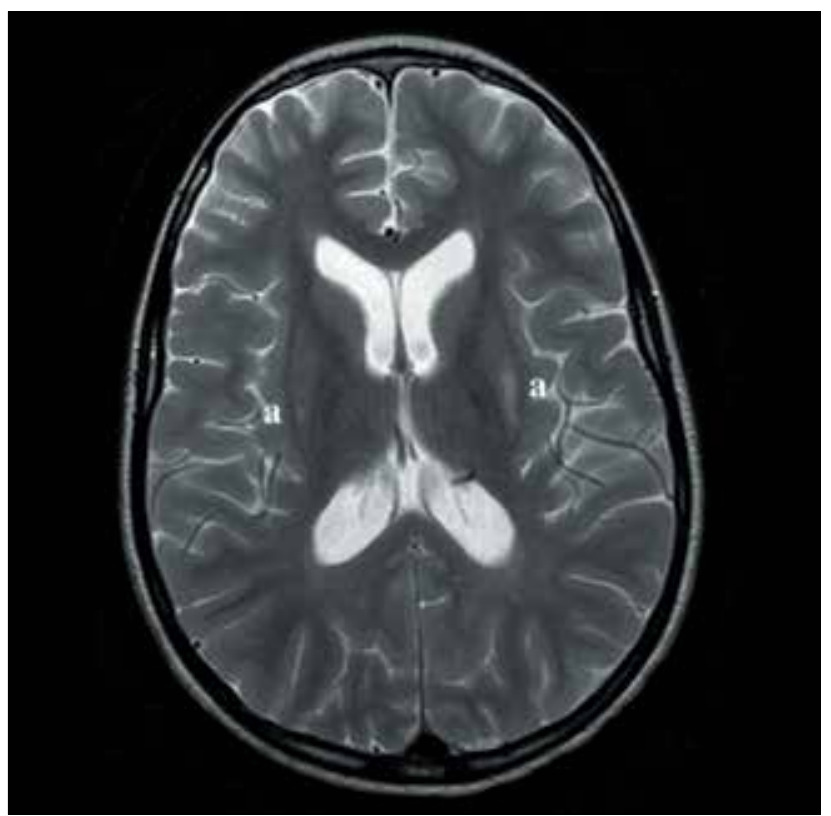

Fig. 1. T2-weighted axial MRI scan of the brain at the level of the basal ganglia. the diagnosis, but elected to remove the child and transferred her into the care of the current caregiver with no explanation of the preceding events hence the precipitant presentation with the misleading history.

A diagnosis of SSPE, stage two, was made and the child was initiated on carbamazepine and isoprinosine. Over the next 12 weeks her condition remained static with persistent myoclonic and atonic seizures. Serial EEGs, while detecting generalised epileptiform discharges, failed to correlate with the clinical events. Her level of awareness continued to fluctuate, but she has not regressed further.

This case is similar to those reported by Kija et al ${ }^{[1]}$ in that the child contracted measles during the 2009 - 2011 epidemic, during the vulnerable period of infancy before her 9-month measles immunisation. While she was 2 years older at presentation than our previous cases, this is more in keeping with the internationally reported cases' presenting age of 5 17 years. ${ }^{[2]}$ Her MRI findings included changes affecting the deep greymatter region, which although unusual have been reported in SSPE. ${ }^{[3,4]}$ To date her clinical deterioration has been slower than in the other cases. ${ }^{[1]}$ It is not known whether this is because she was initiated on isoprinosine relatively early in her course, or because she presented at an older age than the other SA children. As in the other reported cases, her measles PCR was negative, with CSF measles antibody titres confirming the diagnosis.

This additional case demonstrates that SSPE linked to the 2009 2011 measles epidemic continues to occur in the Western Cape region. It is unclear why it has not been reported from other provinces of SA. Clinicians should have a high index of suspicion when a child presents with acquired movement disorders, intractable seizures and cognitive decline, especially those who were infants during the epidemic of 2009 - 2011. Furthermore, the history of measles may not be forthcoming initially, and clinicians may need to pursue this clinical entity in their workup in order to confirm the diagnosis.

\section{Shakti Pillay}

Department of Paediatrics and Child Health, Red Cross War Memorial Children's Hospital and Faculty of Health Sciences, University of Cape Town, South Africa

\section{Ronald van Toorn}

Paediatric Neurology, Tygerberg Hospital, Cape Town, South Africa

\section{Tracy Kilborn}

Department of Radiology, Red Cross War Memorial Children's Hospital, Cape Town, South Africa

\section{Alvin Ndondo}

Paediatric Neurology Unit, Department of Paediatrics and Child Health, Red Cross War Memorial Children's Hospital and Faculty of Health Sciences, University of Cape Town, South Africa

\section{Brian Eley}

Paediatric Infectious Diseases Department, Department of Paediatrics and Child Health, Red Cross War Memorial Children's Hospital and Faculty of Health Sciences, University of Cape Town, South Africa

\section{Jo Wilmshurst}

Paediatric Neurology Unit, Department of Paediatrics and Child Health, Red Cross War Memorial Children's Hospital and Faculty of Health Sciences, University of Cape Town, South Africa jo.wilmshurst@uct.ac.za

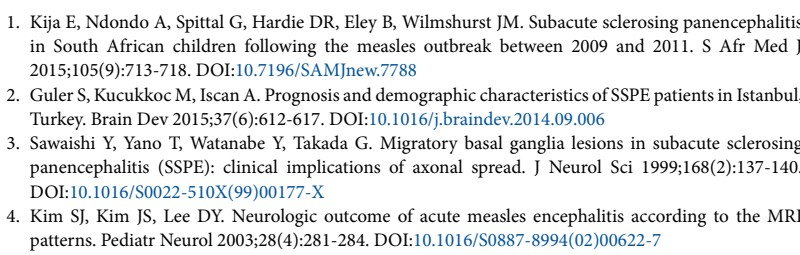
in South African children following the measles outbreak between 2009 and 2011. S Afr Med 2015;105(9):713-718. DOI:10.7196/SAMJnew.7788

2. Guler S, Kucukkoc M, Iscan A. Prognosis and demographic characteristics of SSPE patients in Istanbul, Turkey. Brain Dev 2015;37(6):612-617. DOI:10.1016/j.braindev.2014.09.006

3. Sawaishi Y, Yano T, Watanabe Y, Takada G. Migratory basal ganglia lesions in subacute sclerosing panencephalitis (SSPE): clinical implications of axonal spread. J Neurol Sci 1999;168(2):137-140. DOI:10.1016/S0022-510X(99)00177-X

4. Kim SJ, Kim JS, Lee DY. Neurologic outcome of acute measles encephalitis according to the MR patterns. Pediatr Neurol 2003;28(4):281-284. DOI:10.1016/S0887-8994(02)00622-7

S Afr Med J 2016;106(5):425. DOI:10.7196/SAMJ.2016.v106i5.10630 